\title{
Formation of Two-Dimensional Micelles on Graphene: Multi-Scale Theoretical and Experimental Study
}

Benjamin J. Robinson, ${ }^{*},{ }^{\dagger, \dagger}$ Steven W. D. Bailey, ${ }^{\dagger}$ Luke J. O’Driscoll, ${ }^{\S}$ David Visontai, ${ }^{\dagger, \#}$ Daniel J. Welsh, ${ }^{\S}$ Albertus B. Mostert, ${ }^{\dagger}$ Riccardo Mazzocco, ${ }^{\dagger}$ Caroline Rabot, ${ }^{\|}$Samuel P. Jarvis, ${ }^{\dagger,+, \perp}$ Oleg V. Kolosov, ${ }^{\dagger}$

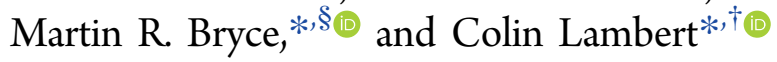

${ }^{\dagger}$ Department of Physics, Lancaster University, Lancaster LA1 4YB, U.K.

${ }^{\ddagger}$ Materials Science Institute, Lancaster University, Lancaster LA1 4YW, U.K.

${ }^{\S}$ Department of Chemistry, Durham University, Durham DH1 3LE, U.K.

"CEA, LETI 17 rue des Martyrs, 38054 Grenoble, France

${ }^{\perp}$ School of Physics and Astronomy, University of Nottingham, Nottingham NG7 2RD, U.K.

\# Department of Physics of Complex Systems, Eötvös University, Pázmány Péter sétány 1/A, H-1117 Budapest, Hungary

\section{Supporting Information}

\begin{abstract}
Graphene and related two-dimensional (2D) materials possess outstanding electronic and mechanical properties, chemical stability, and high surface area. However, to realize graphene's potential for a range of applications in materials science and nanotechnology there is a need to understand and control the interaction of graphene with tailored high-performance surfactants designed to facilitate the preparation, manipulation, and functionalization of new graphene systems. Here we report

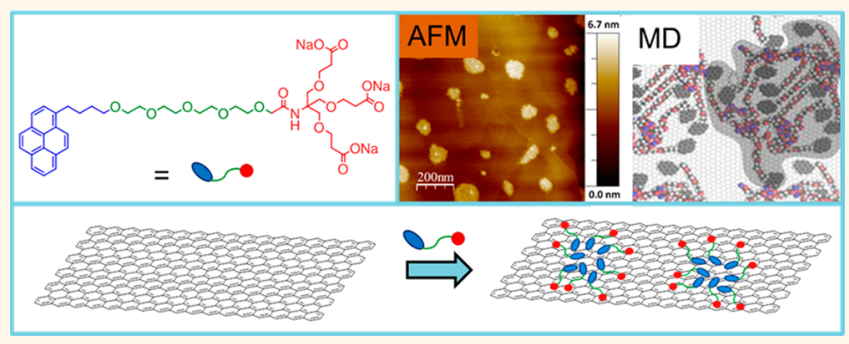
a combined experimental and theoretical study of the surface structure and dynamics on graphene of pyrene-oligoethylene glycol (OEG) -based surfactants, which have previously been shown to disperse carbon nanotubes in water. Molecular self-assembly of the surfactants on graphitic surfaces is experimentally monitored and optimized using a graphene coated quartz crystal microbalance in ambient and vacuum environments. Real-space nanoscale resolution nanomechanical and topographical mapping of submonolayer surfactant coverage, using ultrasonic and atomic force microscopies both in ambient and ultrahigh vacuum, reveals complex, multilength-scale self-assembled structures. Molecular dynamics simulations show that at the nanoscale these structures, on atomically flat graphitic surfaces, are dependent upon the surfactant OEG chain length and are predicted to display a previously unseen class of $2 \mathrm{D}$ self-arranged "starfish" micelles (2DSMs). While three-dimensional micelles are well-known for their widespread uses ranging from microreactors to drug-delivery vehicles, these 2DSMs possess the highly desirable and tunable characteristics of high surface affinity coupled with unimpeded mobility, opening up strategies for processing and functionalizing $2 \mathrm{D}$ materials.
\end{abstract}

KEYWORDS: 2D micelles, surfactants, graphene, scanning probe microscopy, molecular dynamics

urfactants are essential ${ }^{1-4}$ for the efficient separation, dispersion, and functionalization of graphitic materials ${ }^{5}$ in aqueous solution and provide enabling steps in the production of graphene devices. ${ }^{6}$ Surfactants play an essential role in promoting, via ultrasonic or chemically driven exfoliation techniques, ${ }^{2}$ the creation of large graphene flakes with better defined geometries and reduced defect densities ${ }^{7}$ that are vital for the realization of device architectures in fields such as energy storage and generation, ${ }^{8}$ flexible displays, ${ }^{9}$ and sensors. ${ }^{10}$ While the behavior of surfactants in aqueous environments, where they self-assemble into supramolecular assemblies, such as three-dimensional spherical micelles with hydrophobic moieties shielded by a hydrophilic shell, ${ }^{11-13}$ is well understood, their behavior as (sub) monolayers on surfaces has received limited attention. ${ }^{14-16} 3 \mathrm{D}$ micelles may be transferred to solid-liquid or solid-air interfaces resulting in hemispherical or, it has been proposed, disk-like structures, ${ }^{13}$

Received: February 15, 2017

Accepted: March 10, 2017

Published: March 10, 2017 
<smiles>COC(=O)CCOCC(COCCC(=O)O[Na])(COCCC(=O)O[Na])NC(=O)COCCOCCCCc1ccc2ccc3cccc4ccc1c2c34</smiles>

$1(n=2), 2(n=4), 3(n=6)$<smiles>[NH3+]OC(=O)CCOCC(COCCC(=O)O[Na])(COCCC(=O)O[Na])NC(=O)CCCc1ccc2ccc3cccc4ccc1c2c34</smiles>

Figure 1. Left: The structures of the homologous series of surfactants 1-3, which vary only in the length $n$ of the oligoethylene glycol (OEG) linker chain. Right: The structure of surfactant 4, an analogue without an OEG linker. The anchoring unit derived from pyrenebutanol (in 13) or pyrenebutyric acid (in 4) is indicated in blue, the OEG linker unit (in 1-3) is shown in green, and the hydrophilic headgroup based on a first generation Newkome dendron is shown in red.
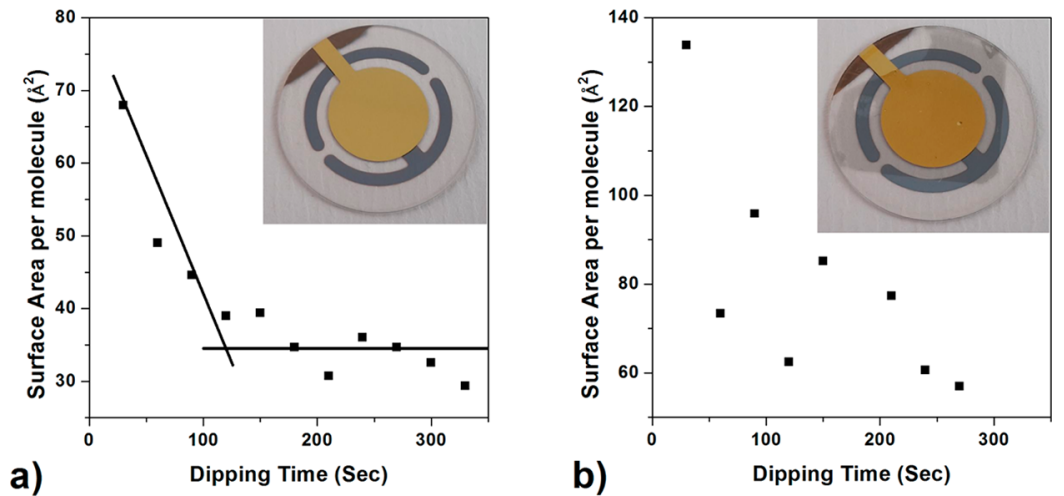

Figure 2. QCM monitored molecular area as a function of the total dipping time of 2 on a $5 \mathrm{MHz}$ QCM with either a (a) gold or (b) graphene surface under ambient condition (inserts: typical Au and graphene coated QCMs).

depending on the interplay between cohesive and adhesive energies, with height in both cases of the same order as the lateral size. However, for sensing and energy storage applications, separation of graphene sheets by surface-stable surfactant structures with a smaller thickness is highly desirable. ${ }^{17,18}$ We now report a combined experimental and theoretical study of the molecular assembly of a tailored class of surfactants on graphitic surfaces. The resulting nano to microscale structuring is shown to derive from the formation of flat $2 \mathrm{D}$ starfish micelles (2DSMs), whose height is much smaller than their lateral surface dimensions. These 2DSMs are not formed by transferring preformed $3 \mathrm{D}$ micelles onto surfaces, but instead arise from the postdeposition aggregation of surfactant molecules on the surface.

\section{RESULTS AND DISCUSSION}

The recently synthesized family of surfactants ${ }^{19}$ (see Figure 1) contain pyrene groups for stable planar anchoring to the graphene surface, connected by oligoethylene glycol (OEG) chains to hydrophilic head groups derived from Newkome dendrons. $^{20}$ Pyrene is known to bind to graphene with a binding energy of $-1.09 \mathrm{eV}$, and to experience a low energy barrier of ca. $0.01 \mathrm{eV}$ to sliding and rotation parallel to the sheet. $^{21-24}$ This results in a desirable combination of (i) strong adsorption of the surfactants onto graphitic surfaces, and (ii) free lateral movement over them. Surfactants 1-4 were synthesized as described previously. ${ }^{19}$

Experimentally, molecular assembly of 2 was monitored by adsorbed molecular layer formation on a quartz crystal microbalance (QCM) sensor surface, on both $\mathrm{Au}$ and graphene, ${ }^{25}$ from aqueous solutions in the concentration range of $0.001-0.1 \mathrm{mg} / \mathrm{mL}$. A Sauerbrey analysis ${ }^{26}$ of the frequency change during assembly was used to determine the area per molecule at frequency plateaus corresponding to stable submonolayer, mono-, and multilayer structures. As pyrene is expected to dominate binding between the surfactants and both $\mathrm{Au}^{27}$ and graphene, ${ }^{28}$ we used the precharacterized $\mathrm{Au}$ coated crystals to benchmark assembly prior to graphene deposition. Molecular concentration and exposure times were modified over several orders of magnitude allowing fine control over the assembly process. Even for the lowest studied concentration of $0.001 \mathrm{mg} / \mathrm{mL}$ and a brief $10 \mathrm{~s}$ deposition, the QCM measurements reveal the presence of added mass in the molecular system due to both surfactant and substrate bound water. While deposition on $\mathrm{Au}$ consistently followed a clear two-stage mechanism (see Figure 2a for example), equivalent conditions resulted in a more stochastic process on graphene (see Figure 2b). This disorder is attributed to the higher surface mobility of the surfactant on graphene ${ }^{24}$ compared to Au. ${ }^{27}$

We were able to differentiate between water bound directly to QCM surface and bound surfactant-structural water (i.e., that associated with the surfactant layer on the surfaces) by analyzing QCM response at the elevated temperatures and vacuum. The QCM response was first calibrated to eliminate temperature dependence of the crystal frequency itself, detailed in SI-1.3. The application of vacuum led to an increase in frequency (indicating decrease in surface mass) of $7.7 \pm 2.2 \mathrm{~Hz}$ and $6.5 \pm 1.5 \mathrm{~Hz}$ for the $\mathrm{Au}$ and graphene systems, respectively. This change in frequency is opposite and smaller than the ca. $30 \mathrm{~Hz}$ frequency decrease (showing mass increase) due to assembly of $\mathbf{2}$ on the surfaces and is independent of the number of vacuum cycles applied. Ab-initio calculations ${ }^{24,29}$ suggest that 

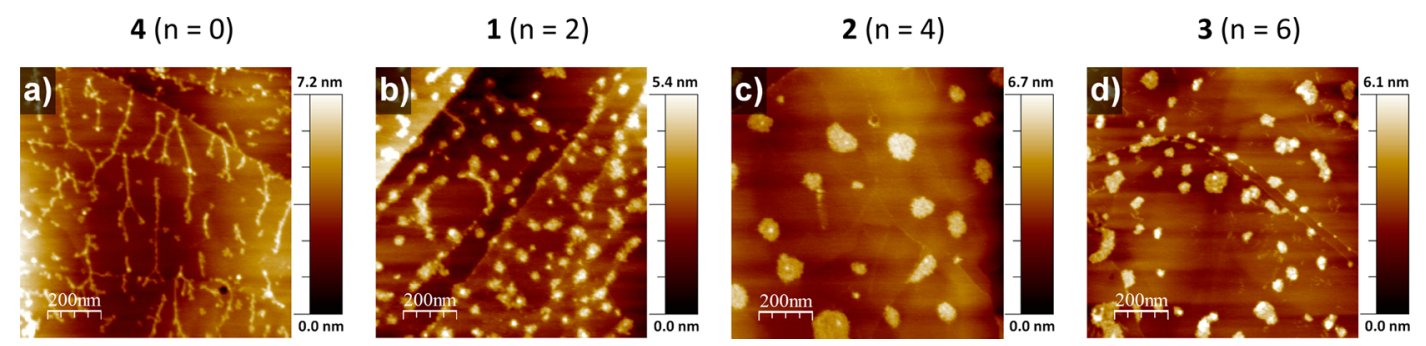

Figure 3. Tapping mode AFM topography images of assembled molecular-structures of surfactants (1-4) on HOPG in ambient conditions where (a) the shortest chain surfactant (4) forms elongated chain-like structures. (b) For 1 a combination of short chain-like and "island" structures are formed. (c) For 2 large, low surface density, islands of the surfactant are formed. (d) For 3 similar island features to 2 are observed. In all cases, at these large scan sizes, section analysis shows that the maximum island height is $<4 \mathrm{~nm}$ which is an order of magnitude less than their typical diameter (see Table 1).

the binding strength of 2 through the pyrene moiety to both $\mathrm{Au}$ and graphene is sufficient to ensure that surfactant molecules remain anchored throughout the vacuum and heating cycles. We therefore ascribe the initial vacuum induced mass decrease to removing of loosely bound physisorbed water on the highly hydrophilic surfactant surface. ${ }^{30}$ Frequency changes arising from $20 \rightarrow 60 \rightarrow 20{ }^{\circ} \mathrm{C}$ heating and cooling cycles under vacuum show a $7 \mathrm{~Hz}$ difference for $\mathrm{Au}$ and a $4 \mathrm{~Hz}$ difference for graphene and, as would be expected without the system being returned to ambient, are again independent of number of heating cycles and indicates all water is removed on the first heat cycle and that no surfactant is removed on subsequent cycles. These changes are assumed to correspond to "structural" water. Based on mass loss calculations we find the removal of 14 water molecules per 2 molecule for Au surfaces and 17 water molecules per 2 molecule for graphene surfaces. If the extra physisorbed water is taken into account, a total of 28 water molecules per 2 molecule for the gold surface and 40 molecules per 2 molecule for the graphene surface are removed. This corresponds with the number of water molecules calculated within our MD simulations, discussed in detail below, suggesting excellent agreement between simulation and experiment for these systems.

\section{MOLECULAR IMAGING}

Direct nanoscale imaging was performed after assembly of surfactant layers on graphene-like freshly cleaved highly ordered pyrolytic graphite (HOPG) surfaces. Intermittent contact (tapping mode) atomic force microscopy (AFM) was used in both ambient and UHV conditions and ultrasonic force microscopy (UFM) $)^{31,32}$ was used in ambient conditions. Freshly cleaved HOPG was exposed to $0.001 \mathrm{mg} / \mathrm{mL}$ solutions of 1-4 for $10 \mathrm{~s}$, rinsed with deionized (DI) water, dried with dry nitrogen, and imaged in ambient conditions (Figure 3 ).

The typical "island" structure of these surface features remains unchanged when the coated HOPG is washed with copious amounts of water and rescanned, confirming the high affinity of the surfactants to the graphene-like surfaces. The features did not appear in control images of HOPG exposed to DI water alone. Although the structures formed vary significantly between surfactants, there are several consistent features. First, the height profiles of all "islands" are typically $<4$ $\mathrm{nm}$, which is small relative to the lateral dimension of the features, and, second, there are indications of internal structure within these $2 \mathrm{D}$ features. Finally, these island features are of very different length scales to the $3 \mathrm{D}$ micelles observed in bulk solution as shown in Table 1 below (see also SI-3).
Table 1. Typical Diameters of Circular Features Formation by Surfactants 1-3 As Observed via Tapping AFM on the HOPG Surface and via DLS in Bulk

\begin{tabular}{ccc} 
surfactant & $\begin{array}{c}\text { typical 2D dimension from } \\
\text { AFM (nm) }\end{array}$ & $\begin{array}{c}\text { typical 3D dimension from } \\
\text { DLS (nm) }\end{array}$ \\
$\mathbf{1}$ & $44.65 \pm 11.60$ & $384 \pm 4$ \\
$\mathbf{2}$ & $54.44 \pm 25.50$ & $435 \pm 4$ \\
3 & $46.43 \pm 18.09$ & $498 \pm 3$ \\
\hline
\end{tabular}

This fine structure within the islands was subsequently investigated using UFM and small area tapping mode topography, shown in Figure $4 \mathrm{~d}$ and e, respectively. UFM eliminates the friction due to vertical ultrasonic oscillation of the sample, ${ }^{33}$ while preserving the nanoscale nanomechanical contrast to both stiff and soft objects. ${ }^{32,34}$

The UFM scan in Figure $4 \mathrm{~b}$ shows the compliant (darker contrast) areas corresponding to higher protrusions observed in contact mode topography (Figure 4a)-typical for UFM nanomechanical contrast of small organic systems. ${ }^{35}$ The area shows a surface region of high molecular coverage-the HOPG substrate can be seen on the right-hand edge of the image, indicated by circular green dashes. The small scale $(120 \times 120$ $\mathrm{nm}$ ) tapping mode scan of Figure $4 \mathrm{e}$ shows distinct populations within the surfactant structures; with height distribution analysis (Figure 4f) indicating typical heights of these features at $0.60 \pm 0.21 \mathrm{~nm}$ (with typical diameters obtained by section analysis of $12.79 \pm 2.93 \mathrm{~nm}$ ) and lower frequency higher features in the range $1.1-1.4 \mathrm{~nm}(16.77 \pm 1.99 \mathrm{~nm}$ diameter $)$, matching well with the UFM nanomechanical maps. Blurring and step-like shifts in the fast scan (horizontal) axis in Figure 4e are due to tip-induced disruption of the film, despite the extremely small lateral forces inherent to tapping mode AFM. This is consistent with the high lateral mobility of the surfactants on the substrate which also precluded clear imaging with scanning tunneling microscopy (STM) even in UHV at 77 $\mathrm{K}$. Some elongation in the slow (vertical) scan axis is believed to be due to thermal drift arising from the slow scan speeds necessary for tapping mode imaging of soft objects. Comparable imaging of $\mathbf{2}$ was performed in the absence of surface water (Figure 5) by imaging in UHV at $77 \mathrm{~K}$. Sample preparation for UHV measurements followed exactly the same protocol as the ambient samples, with only the addition of a further annealing step under vacuum $\left(50^{\circ} \mathrm{C}\right.$ for $\left.\sim 11 \mathrm{~h}\right)$ before being introduced into the main scan chamber. As with ambient scanning conditions, multilength scale structures were observed with smallest feature sizes corresponding to lateral dimensions of $\sim 10 \mathrm{~nm}$ (Figure 5, line profile 2) and a height of 1-2 nm. In 

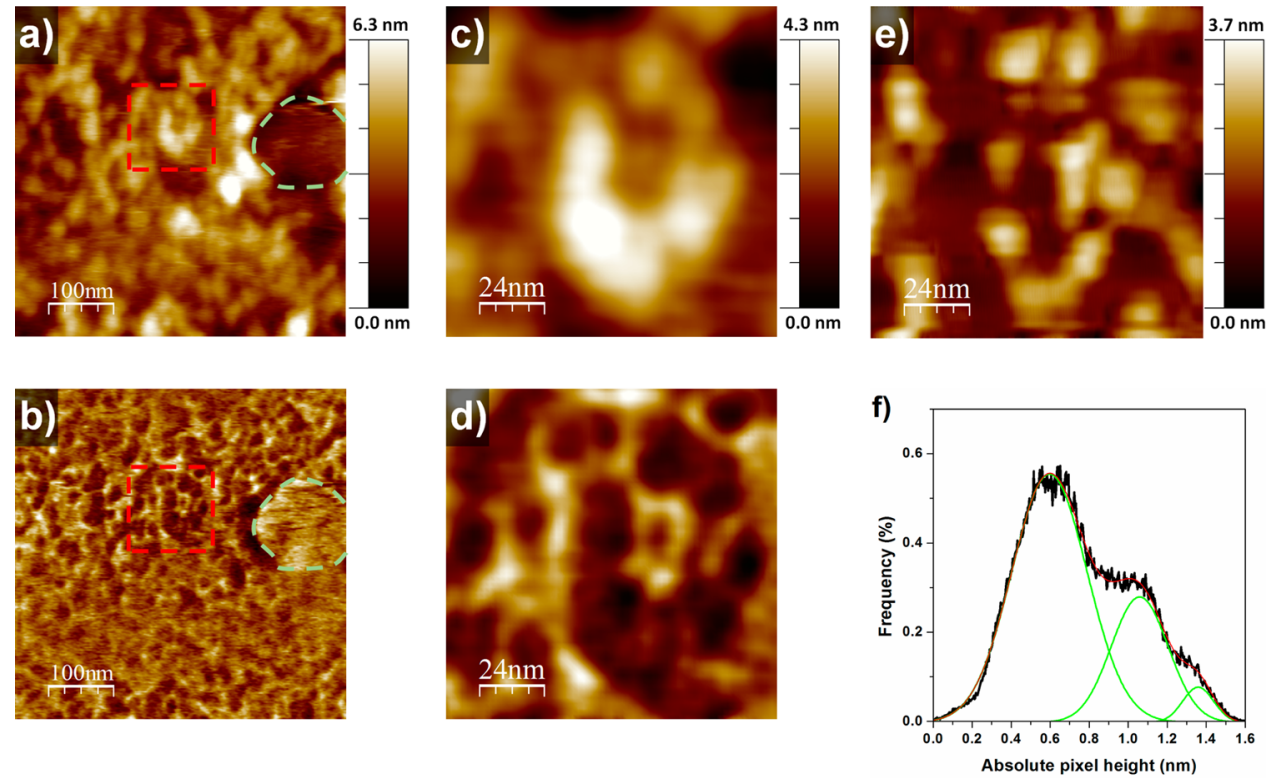

Figure 4. High-resolution SPM imaging of surfactant nanostructure in ambient conditions showing (a) contact mode AFM topography and in (b) the simultaneously acquired UFM nanomechanical response (where darker areas correspond to lower stiffness) of a densely packed film of 2 on HOPG. Topography image (c) and corresponding UFM image in (d) show zoomed-in details of molecular clusters shown within red dashed boxes in (a) and (b), respectively. (e) The small scan size tapping mode topography of 2 on HOPG and (f) the corresponding image height distribution histogram. Exposed areas of HOPG, indicated with a green dashed line, are clearly visible in both the topography and UFM nanomechanical images.
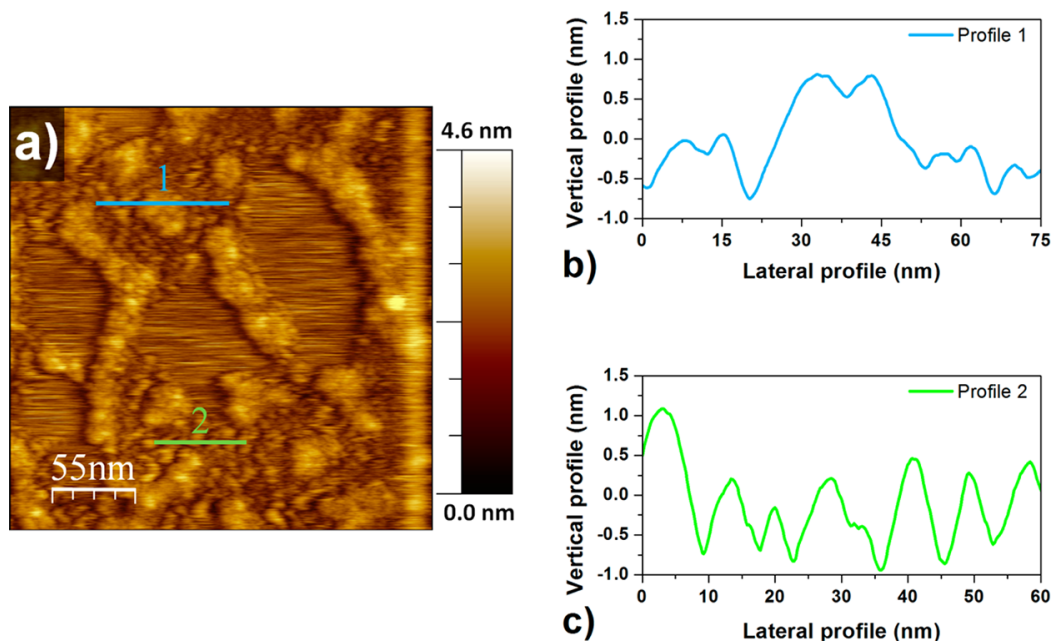

Figure 5. High-resolution frequency modulation FM-AFM imaging under UHV conditions with a qPlus sensor. (a) Constant $\Delta f$ feedback image showing mono and bilayer molecular structures. Scan size: $250 \times 250 \mathrm{~nm}, \Delta f$ set point $=+10 \mathrm{~Hz}, a_{0}=225 \mathrm{pm}, f_{0}=23.7 \mathrm{kHz}$. Line profiles show (b) a molecular height profile varying from $\sim 1-2 \mathrm{~nm}$ and (c) smallest lateral feature sizes of $\sim 10 \mathrm{~nm}$.

the UHV measurements a second layer structure was also observed confirming a layer height of $\sim 1 \mathrm{~nm}$ between the first and second molecular layers (Figure 5, line profile 1). This removes any possibility that the molecular layer appears with a lower height due to possible compression by the AFM tip compared to the practically incompressible HOPG surface.

\section{DSM SIMULATIONS}

To understand the nature of the observed structures, in both ambient and UHV conditions, we performed molecular dynamic (MD) simulations (see S4-1-3 for details), initially investigating the assembly of the surfactants on a disordered graphene sheet both in the absence and presence of water. In both cases the nonbonded $\mathrm{Na}^{+}$counterions of the hydrophilic headgroup were free to escape and recoordinate with the three carboxylate moieties during the simulation; however, in the absence of water, none were observed to do so. Upon introduction of water the $\mathrm{Na}^{+}$counterions were able to escape from the vicinity of the headgroup (see SI). Figure 6 shows the resultant structures for surfactants 1,2 , and 3. The first six snapshots (Figure 6a-f) show that for all three surfactants, in the absence of water, structures form in which the hydrophilic charge-neutral Newkome Dendron headgroups aggregate via van der Waals forces in the interior with the hydrophobic pyrene anchors on the exterior. However, upon the introduction of water to the simulation, as shown in panels g-i of Figure 6, the hydrophobic pyrene anchors flip from the exterior to the interior of the structures, where they aggregate 


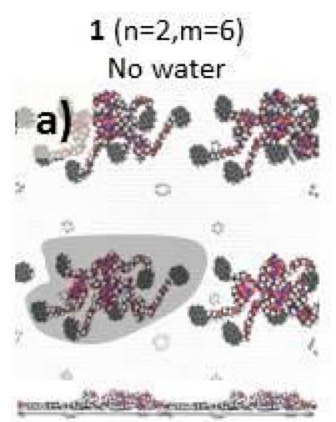

$1(\mathrm{n}=2, \mathrm{~m}=12)$

No water

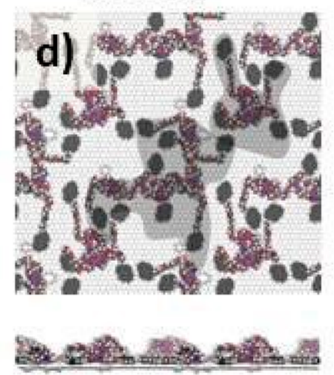

$1(n=2, m=6)$

With water

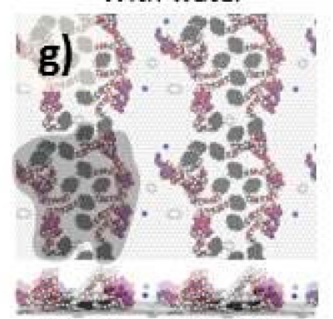

$2(\mathrm{n}=4, \mathrm{~m}=6)$

No water

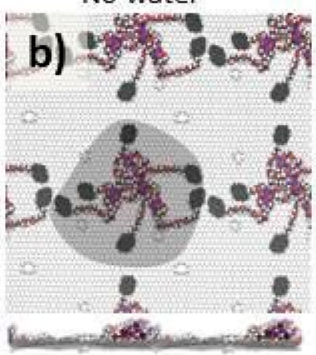

$2(\mathrm{n}=4, \mathrm{~m}=12)$

No water

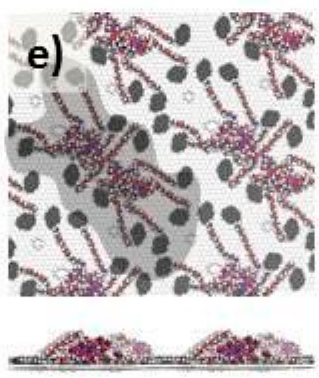

$2(\mathrm{n}=4, \mathrm{~m}=6)$

With water

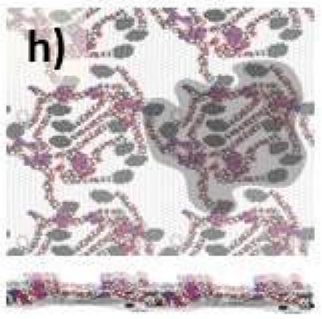

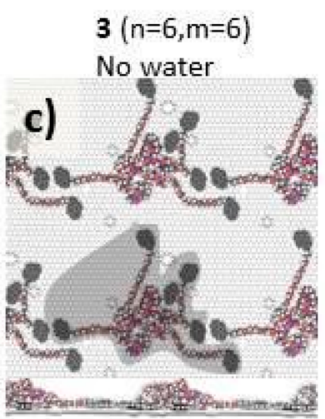

$3(n=6, m=12)$

No water

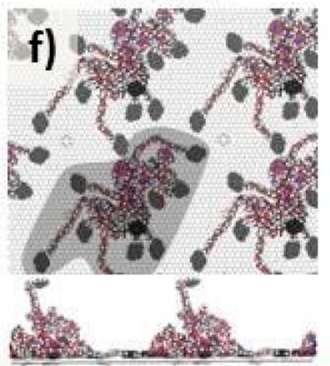

$3(n=6, m=12)$

With water

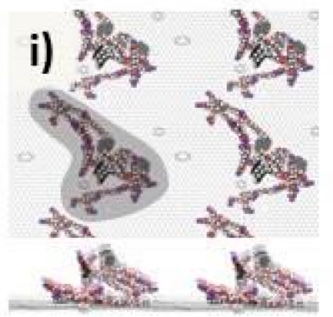

Figure 6. MD simulations of 2DSM assembly formed by surfactants 1,2 , and 3 (with chain lengths $n=2$, 4, and 6 , respectively, and containing $m=6$ or $m=12$ surfactant molecules) on a $42.58 \mathrm{~nm}^{2}$ defective graphene surface at $300 \mathrm{~K}$ and 1 atm. Panels a-f show snapshots of the $2 \mathrm{DSM}$ assembly in the absence of water and panels $\mathrm{g}-\mathrm{i}$ show snapshots in the presence of water. The snapshots show four periodic duplicate cells of the graphene sheet with the water not shown for clarity. The integer $m$ denotes the number of surfactant molecules/42.58 nm ${ }^{2}$ sheet. The sheet carbon atoms are shown in light gray with other carbons in dark gray/black, oxygen in red, hydrogen in white, nitrogen in purple, and sodium in blue.

by van der Waals forces and are surrounded by the OEG linkers and headgroups. In this case, the Newkome dendrons can extend into the water layers above the graphene. This is a $2 \mathrm{D}$ analogue of the well-known spherical micelle. These 2D micelles possess a "starfish-like" structure (2DSM) with thicknesses 2 orders of magnitude smaller than their typical lateral diameter corresponding with the dimensions of the features observed by SPM imaging. In the MD simulations at low coverage of surfactant $\mathbf{1}$ in water (see S4: Cluster Sizes) the surfactant has a lateral diameter of $43.8 \AA$ with an extension from the graphene surface of $8 \AA$. The lateral diameter increases with the surfactant concentration and levels out at $56.4 \AA$ at the maximum surface coverage predicted to occur when $m=15$. At higher concentrations the surfactants cluster to form conical structures. See S4-4: Cluster Sizes for details. Starfish 2DSMs are distinct from conventional micelles or lipid membranes ${ }^{36}$ as surface interactions and topological steric hindrance, determined by the length of the OEG bridges separating the hydrophobic and hydrophilic moieties, play a major role in determining their dynamics and shapes, as do their local environment. One such environmental effect can be seen in the snapshot shown in Figure 6 panel $\mathrm{g}$ for surfactant 1 (chain length $n=2$ and density $m=6$ molecules), which shows examples of $\mathrm{Na}^{+}$ions (colored blue) both coordinated to the carboxylate moieties and dissociated into the water. As a crosscheck to the robustness of the prediction of $2 \mathrm{D}$ micelle formation the graphene sheet was increased 4-fold so that the simulation area is increased to $170.32 \mathrm{~nm}^{2}$. This avoids periodic effects as the lower concentrations now do not interact between unit cells. There was no change to the quantitative observation of 2D micelle formation (see SI S4.2).

The details of the distribution of the $\mathrm{Na}^{+}$ions are revealed by the pair correlation function for the separation of $\mathrm{Na}^{+}$and the $\mathrm{O}^{-}$ions in the carboxylate $\left(\mathrm{COO}^{-}\right)$moieties. Figure 7 for surfactant 2 compares the pair correlation function in the absence and presence of water. Clearly in the absence of water (shown by the black curve) the $\mathrm{Na}^{+}$ions are coordinated to the $\mathrm{O}^{-}$ion in the $\left(\mathrm{COO}^{-}\right)$moiety at a separation of $2.5 \AA$ and also associated with the neighboring carboxylate moieties at a separation of $\sim 5.0 \AA$. In the presence of water (shown by the red curve) the $\mathrm{Na}^{+}$ions spend much less time coordinated with an $\mathrm{O}^{-}$ion and are more randomly distributed over the 


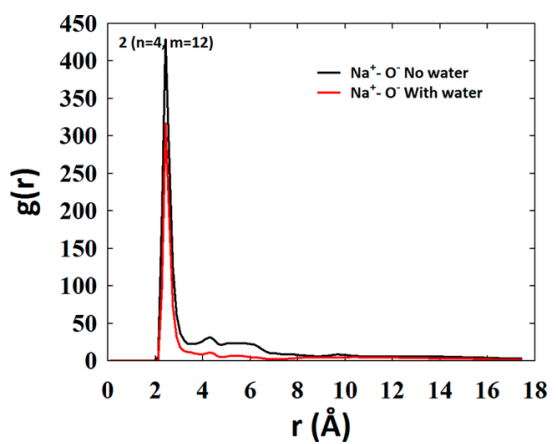

Figure 7. The pair correlation function over the production run of the simulation for the surfactant 2 is shown. In the absence of water (shown by the black curve) the $\mathrm{Na}^{+}$ions are coordinated to the $\mathrm{O}^{-}$ ion in the $\left(\mathrm{COO}^{-}\right)$moiety at a separation of $2.5 \AA$ and also associated with the neighboring carboxylate moieties at a separation of $\sim 5.0 \AA$. In the presence of water (shown by the red curve) the $\mathrm{Na}^{+}$ions spend much less time coordinated with an $\mathrm{O}^{-}$ ion and are more randomly distributed over the simulation cell.

simulation cell. This result is also demonstrated in Figure S10 for surfactant 1. The anchor units remain strongly adsorbed onto the graphene surface; however, in the presence of water, as the $\mathrm{Na}^{+}$ions leave the initially charge-neutral headgroups, the anionic dendrons repel each other and are attracted into the lower layers of water by van der Waals forces. This effect can be seen by comparing Figure S9 panel 3 where at a high concentration of surfactant $\mathbf{2}$ in the absence of water the molecules spread out over the surface but in the presence of water in panel 6 conical structures form

The structures of the 2DSMs depend not only upon the length $n$ of the OEG linker, but also the coverage, which we quantify by the number $m$ of molecules on the $42.58 \mathrm{~nm}^{2}$ sheet. For example as $m$ increases from 6 to 12 surfactant 1 tends to form linear structures, whereas surfactant 2 forms a 12 legged 2DSM, and surfactant 3 starts to develop a conical structure as some of the headgroups and the pyrene anchor units extend away from the graphene surface. Increasing $m$ to 24 for surfactant 2 (shown in Figure S9 panels 1-3) results in 12 of the pyrene anchors forming a base which further molecules stack on top of. Thus, the graphene surface remains only partially covered and complete surface coverage is not observed. The trends observed for the surfactants in the absence of water in Figure 6 are very similar to those observed in the presence of water. Surfactant 1 tends to form 2D linear micelles but in this case with the pyrene units sandwiched between the hydrophilic units. Surfactant 2 forms a more circular 12 legged 2DSM as the hydrophilic groups surround the pyrene units. Surfactant 3 again starts to develop a conical structure as some of the head groups and pyrene anchor units extend away from the graphene surface. Figure S9 panels 4-6 for surfactant 2 with $m=24$ in the presence of water also shows that 12 of the pyrene anchors form a base with further molecules stacking on top. This again results in incomplete surface coverage and as the cone extends into the water layers we can estimate the number of water molecules associated with the surfactant outer layers as approximately 40 per molecule of 2. This is in excellent agreement with the number measured by QCM, above.

\section{CONCLUSIONS}

The assembly and behavior of a series of tailored surfactant molecules on graphitic surfaces has been investigated in both ambient and high vacuum conditions. In both cases multilength scale surfactant features are observed with the smallest observable feature possessing a near 2D profile. Assembly of such $2 \mathrm{D}$ structures is supported by MD simulations; furthermore $\mathrm{MD}$ calculated the same ratio of surfactant to water molecules on the graphene surface as direct QCM quantitative molecular adsorption experiments. MD confirms that these $2 \mathrm{D}$ structures behave as planar micelles on the surface, with a structure dependent on the presence or absence of water. MD also shows that the strongly surface-attached pyrene anchor units are located at the periphery of the structures in the absence of water, but at the core in the presence of water. The existence and manipulation of 2DSMs on graphene will be important for the control of surface functionalization and a range of associated technologies. The realization of 2DSM opens routes to developing 2D microreactors which could lead to important advances, including control of the size and shape of 2D nanoislands grown on surfaces, with applications to nanoscale circuitry and biosensing. The chemical tunability of surfactants could enable the engineering of tailored structural molecular features in multifunctional graphene-based materials. Furthermore, the ability to use scanning probe technologies to image 2DSMs in solution is likely to have ramifications for our understanding of the fundamental science of micelle formation and control. A mechanism for noncovalent molecular capture can be postulated where a molecule in solution is captured noncovalently by van der Waals forces within the Newkome dendrons and as the surfactant is dried so the molecule is held as the micelle reverses. Unwanted ions in solution can also exchange with the $\mathrm{Na}^{+}$ions associated with the carboxylate moieties and can be removed after drying. If the surfactants can be repeatedly dried and rehydrated the captured molecules or ions could then be repeatedly captured and released. The tendency for surfactant 3 to extend into the water environment can be exploited as a device design mechanism. A layer of the surfactant on graphene forms conical structures and depositing another graphene layer over the cones would result in the two graphene layers being separated by discrete pillars. This could have applications for thermoelectrics as the soft micelle pillars would inhibit phonon transport across the graphene layers.

\section{METHODS}

Quartz Crystal Microbalance. Segregation grown graphene ${ }^{37}$ was transferred onto the top Au electrode of the QCM liquid handling head resulting in films of 1-2 graphene layers thickness, with continuous coverage over the entire QCM electrode area resulting in maximal sensitivity (more details in SI-1). ${ }^{38}$ QCMs were dip-coated in surfactant solution, in the range $0.001-0.1 \mathrm{mg} / \mathrm{mL}$ for $10 \mathrm{~s}$, prior to washing with DI water and nitrogen drying. The QCM frequency was measured and then the process repeated. No change in frequency was observed for the subsequent DI water washing/dry nitrogen drying stages suggesting these steps did not remove the surfactant. A custom vacuum chamber comprising a high thermal conductivity aluminum base in a direct thermal contact with a large area Peltier heater/cooler was used to ensure rapid thermal equilibrium in the system, further details are given in SI-1.3. A relevant temperature range of $20-60{ }^{\circ} \mathrm{C}$ was used with a pressure varied alternated between ambient and $10^{-1}$ Torr. Continuous vacuum pumping ensures that water removed by heating was efficiently evacuated from the chamber. Both Au and graphene QCMs were calibrated for temperature induced frequency 
changes; the frequency changes solely due to water evaporation as a function of vacuum and temperature on graphene are given in Figure S3.

Ambient SPM Imaging. Contact mode, tapping mode atomic force microscopy and ultrasonic force microscopy were all performed using a Multimode Nanoscope IV AFM (Bruker AXS). For tapping mode high stiffness $k=40 \mathrm{~N} \mathrm{~m}^{-1}$ and $300 \mathrm{kHz}$ resonance (Tap300-G, Budget Sensors) probes were used. Topography and phase (shown in SI) were captured simultaneously. As UFM is essentially a modification of contact mode imaging albeit with eliminated friction, probes with $k=0.2 \mathrm{~N} \mathrm{~m}^{-1}$ (Contact-G, Budget Sensors) were used. A thin plate piezoceramic actuator $(4 \mathrm{MHz}$ thickness mode resonance, PI) was bonded to the sample substrate and used to generate ultrasonic vibrations, resulting in efficient ultrasonic excitation at frequencies up to $10 \mathrm{MHz} .^{32}$ The piezoceramic actuator was excited by a function generator (LXI Keithley) at $4.2 \mathrm{MHz}$ ultrasonic frequency modulated at $2.3 \mathrm{kHz}$; the cantilever deflection at this modulation frequency corresponds to the nonlinear UFM response, which is highly dependent on the local nanomechanical properties of the sample, and was detected via a lock-in amplifier (Stanford Research Systems, SRS-830) as described in detail elsewhere. ${ }^{39,40}$

High-Vacuum SPM Imaging. Frequency modulation atomic force microscopy (FM-AFM) measurements collected under ultrahigh vacuum (UHV) were obtained using an Omicron Nanotechnology GmbH LT STM-AFM system operating at liquid nitrogen temperature $(77 \mathrm{~K})$ and an Omicron Nanotechnology GmbH VT STM-AFM operating at room temperature. Both systems were kept under ultrahigh vacuum conditions with a base pressure of $5 \times 10^{-11} \mathrm{mbar}$ or better. FM-AFM measurements at LT were collected using a commercial qPlus sensor ${ }^{41}$ supplied by Omicron $\mathrm{GmbH}$ with a resonant frequency of $\sim 23.7 \mathrm{kHz}$ and a stiffness of $\sim 2000 \mathrm{~N} \mathrm{~m}^{-1}$. For all FM-AFM measurements images were acquired under feedback by maintaining a constant $\Delta f$ set point. Prior to all measurements on the micelle samples, images were collected on clean $\mathrm{Ag}(111)$ surfaces where the tip was conditioned (via standard voltage pulsing and tipindentation methods) until atomically sharp steps were observed, thus confirming the presence of an atomically sharp probe. We have therefore neglected any contribution of tip dilation from quoted dimensions for these measurements. Unusually, imaging in the conventional attractive mode (i.e., maintaining a negative value of $\Delta f$ ) was found to be exceptionally challenging and snap-to-contact was often observed. We therefore instead performed the majority of measurements using positive $\Delta f$ set points where images were found to be more stable.

Molecular Modeling. Molecular dynamic (MD) simulations were performed to investigate the assembly of the surfactants on the surface of a disordered graphene sheet in water. The simulations were computed by using the MD package DLPOLY $4 .{ }^{42}$ The system was modeled as a periodic cuboid of water (parametrized to the TIP3P water model at $300 \mathrm{~K}, 1 \mathrm{~atm}$ and a density of $1000 \mathrm{~kg} / \mathrm{m}^{3}$ ) of side 60 $\times 60 \times 75 \AA$ A which contained a number, $m$, of surfactant units placed over a periodic, disordered sheet of graphene of size $60 \times 75 \AA$. Each calculation was relaxed for $8 \mathrm{~ns}$ prior to obtaining the snapshots shown in Figure 6 of the main text using the Nosé-Hoover (npt) thermostat with barostat ensemble. An adapted force field was constructed using the Dreiding force field scheme with the parameters found in Mayo et al. ${ }^{43}$ and charges allocated to each atomic species calculated by the DFT package SIESTA ${ }^{44}$ using the van der Waals density functional ${ }^{45-47}$ where extended double- $\zeta$ polarized basis sets of the pseudo atomic orbitals were used to optimize the geometries by relaxing the atomic forces to less than $20 \mathrm{meV} / \mathrm{A}$. Importantly, the force field charges were carefully tuned to allow the $\mathrm{Na}^{+}$ions coordinated to the three carboxylate moieties in the headgroup shown in Figure 1, to disperse into the water environment and recombine during the simulation. The overall system charge was neutral.

\section{ASSOCIATED CONTENT}

\section{Supporting Information}

The Supporting Information is available free of charge on the ACS Publications website at DOI: 10.1021/acsnano.7b01071.

Details of quartz crystal microbalance measurements, additional SPM imaging, details of dynamic light scattering studies of 3D micelle structures, and extensive details of molecular modeling (PDF)

Movie of the quantitative observation of $2 \mathrm{D}$ micelle formation for surfactant 2 with $m=12$ in the absence of water (AVI)

Movie of formation of two 2D micelles placed on the surface and then allowed to interact (AVI)

Movie of surfactant $\mathbf{1}(n=2, m=6)$ in the absence of water (AVI)

Movie of surfactant $2(n=4, m=6)$ in the absence of water (AVI)

\section{AUTHOR INFORMATION}

\section{Corresponding Authors}

*E-mail: b.j.robinson@lancaster.ac.uk.

*E-mail: m.r.bryce@durham.ac.uk.

*E-mail: c.lambert@lancaster.ac.uk.

ORCID

Benjamin J. Robinson: 0000-0001-8676-6469

Martin R. Bryce: 0000-0003-2097-7823

Colin Lambert: 0000-0003-2332-9610

\section{Notes}

The authors declare no competing financial interest.

\section{ACKNOWLEDGMENTS}

We thank BP Exploration Operating Company Ltd. for funding the chemical synthesis and the MD calculations, and $\mathrm{Dr} \mathrm{H}$. Frampton and Dr D. Chappell for helpful discussions. We thank EU FP7 grants GRENADA (GA-246073) and QUANTIHEAT (GA- 604668) for funding the experimental work at Lancaster, we thank EPSRC grant EP/K0394/23/1 for funding characterization work at Durham and Professor Ian Gentle (University of Queensland) and Professor Philip Moriarty (University of Nottingham) for helpful discussions. S.P. Jarvis would like to thank the Leverhulme Trust for fellowship ECF-2015-005.

\section{REFERENCES}

(1) Novoselov, K. S.; Fal'ko, V. I.; Colombo, L.; Gellert, P. R.; Schwab, M. G.; Kim, K. A Roadmap for Graphene. Nature 2012, 490, 192-200.

(2) Soldano, C.; Mahmood, A.; Dujardin, E. Production, Properties and Potential of Graphene. Carbon 2010, 48, 2127-2150.

(3) Notley, S. M. Highly Concentrated Aqueous Suspensions of Graphene through Ultrasonic Exfoliation with Continuous Surfactant Addition. Langmuir 2012, 28, 14110-14113.

(4) Rodriguez-Perez, L.; Herranz, M. a. A.; Martin, N. The Chemistry of Pristine Graphene. Chem. Commun. 2013, 49, 3721-3735.

(5) Novoselov, K. S.; Geim, A. K.; Morozov, S. V.; Jiang, D.; Zhang, Y.; Dubonos, S. V.; Grigorieva, I. V.; Firsov, A. A. Electric Field Effect in Atomically Thin Carbon Films. Science 2004, 306, 666-669.

(6) Lotya, M.; Hernandez, Y.; King, P. J.; Smith, R. J.; Nicolosi, V.; Karlsson, L. S.; Blighe, F. M.; De, S.; Wang, Z.; McGovern, I. T.; Duesberg, G. S.; Coleman, J. N. Liquid Phase Production of Graphene by Exfoliation of Graphite in Surfactant/Water Solutions. J. Am. Chem. Soc. 2009, 131, 3611-3620. 
(7) Hassan, M.; Reddy, K. R.; Haque, E.; Minett, A. I.; Gomes, V. G. High-Yield Aqueous Phase Exfoliation of Graphene for Facile Nanocomposite Synthesis Via Emulsion Polymerization. J. Colloid Interface Sci. 2013, 410, 43-51.

(8) Choi, H. J.; Jung, S. M.; Seo, J. M.; Chang, D. W.; Dai, L. M.; Baek, J. B. Graphene for Energy Conversion and Storage in Fuel Cells and Supercapacitors. Nano Energy 2012, 1, 534-551.

(9) Wu, J.; Agrawal, M.; Becerril, H. A.; Bao, Z.; Liu, Z.; Chen, Y.; Peumans, P. Organic Light-Emitting Diodes on Solution-Processed Graphene Transparent Electrodes. ACS Nano 2010, 4, 43-48.

(10) He, Q.; Wu, S.; Yin, Z.; Zhang, H. Graphene-Based Electronic Sensors. Chem. Sci. 2012, 3, 1764-1772.

(11) Hsieh, A. G.; Korkut, S.; Punckt, C.; Aksay, I. A. Dispersion Stability of Functionalized Graphene in Aqueous Sodium Dodecyl Sulfate Solutions. Langmuir 2013, 29, 14831-14838.

(12) Glover, A. J.; Adamson, D. H.; Schniepp, H. C. Charge-Driven Selective Adsorption of Sodium Dodecyl Sulfate on Graphene Oxide Visualized by Atomic Force Microscopy. J. Phys. Chem. C 2012, 116, 20080-20085.

(13) Israelachvili, J. Self-Assembly in Two Dimensions: Surface Micelles and Domain Formation in Monolayers. Langmuir 1994, 10, 3774-3781.

(14) Greenwood, J.; Phan, T. H.; Fujita, Y.; Li, Z.; Ivasenko, O.; Vanderlinden, W.; Van Gorp, H.; Frederickx, W.; Lu, G.; Tahara, K.; Tobe, Y.; Uji-i, H.; Mertens, S. F. L.; De Feyter, S. Covalent Modification of Graphene and Graphite Using Diazonium Chemistry: Tunable Grafting and Nanomanipulation. ACS Nano 2015, 9, 55205535.

(15) Hsieh, A. G.; Punckt, C.; Korkut, S.; Aksay, I. A. Adsorption of Sodium Dodecyl Sulfate on Functionalized Graphene Measured by Conductometric Titration. J. Phys. Chem. B 2013, 117, 7950-7958.

(16) Xu, S.-L.; Wang, C.; Zeng, Q.-D.; Wu, P.; Wang, Z.-G.; Yan, H.K.; Bai, C.-L. Self-Assembly of Cationic Surfactants on a Graphite Surface Studied by Stm. Langmuir 2002, 18, 657-660.

(17) Green, A. A.; Hersam, M. C. Emerging Methods for Producing Monodisperse Graphene Dispersions. J. Phys. Chem. Lett. 2010, 1, 544-549.

(18) Choi, I.; Kulkarni, D. D.; Xu, W.; Tsitsilianis, C.; Tsukruk, V. V. Star Polymer Unimicelles on Graphene Oxide Flakes. Langmuir 2013, 29, 9761-9769.

(19) Welsh, D. J.; O’Driscoll, L. J.; Bailey, S. W. D.; Visontai, D.; Howes, K.; Frampton, H.; Bryce, M. R.; Lambert, C. J. Key Role of the Linker in Pyrene-Linker-Carboxylate Surfactants for the Efficient Aqueous Dispersion of Multiwalled Carbon Nanotubes. RSC Adv. 2015, 5, 95360-95368.

(20) Newkome, G. R.; Behera, R. K.; Moorefield, C. N.; Baker, G. R. Chemistry of Micelles. 18. Cascade Polymers: Syntheses and Characterization of One-Directional Arborols Based on Adamantane. J. Org. Chem. 1991, 56, 7162-7167.

(21) Zhang, Y.; Liu, C.; Shi, W.; Wang, Z.; Dai, L.; Zhang, X. Direct Measurements of the Interaction between Pyrene and Graphite in Aqueous Media by Single Molecule Force Spectroscopy: Understanding the П-П Interactions. Langmuir 2007, 23, 7911-7915.

(22) Liu, J.; Bibari, O.; Mailley, P.; Dijon, J.; Rouviere, E.; SauterStarace, F.; Caillat, P.; Vinet, F.; Marchand, G. Stable Non-Covalent Functionalisation of Multi-Walled Carbon Nanotubes by PyrenePolyethylene Glycol through П-П Stacking. New J. Chem. 2009, 33, 1017-1024.

(23) Backes, C.; Mundloch, U.; Ebel, A.; Hauke, F.; Hirsch, A. Dispersion of Hipco ${ }^{\circledR}$ and Comocat ${ }^{\circledR}$ Single-Walled Nanotubes (Swnts) by Water Soluble Pyrene Derivatives-Depletion of Small Diameter Swnts. Chem. - Eur. J. 2010, 16, 3314-3317.

(24) Bailey, S.; Visontai, D.; Lambert, C. J.; Bryce, M. R.; Frampton, H.; Chappell, D. A Study of Planar Anchor Groups for GrapheneBased Single-Molecule Electronics. J. Chem. Phys. 2014, 140, 054708.

(25) Zhihua, Y.; Liang, Z.; Kaixin, S.; Weiwei, H. Characterization of Quartz Crystal Microbalance Sensors Coated with Graphene Films. Procedia Eng. 2012, 29, 2448-2452.
(26) Sauerbrey, G. Use of Quartz Vibration for Weighing Thin Films on a Microbalance. Phys. J. 1959, 155, 206-222.

(27) Pham, T. A.; Song, F.; Nguyen, M.-T.; Stohr, M. Self-Assembly of Pyrene Derivatives on $\mathrm{Au}(111)$ : Substituent Effects on Intermolecular Interactions. Chem. Commun. 2014, 50, 14089.

(28) Anne, A.; Bahri, M. A.; Chovin, A.; Demaille, C.; Taofifenua, C. Probing the Conformation and 2d-Distribution of Pyrene-Terminated Redox-Labeled Poly(Ethylene Glycol) Chains End-Adsorbed on Hopg Using Cyclic Voltammetry and Atomic Force Electrochemical Microscopy. Phys. Chem. Chem. Phys. 2014, 16, 4642-4652.

(29) Medeiros, P. V. C.; Gueorguiev, G. K.; Stafström, S. Benzene, Coronene, and Circumcoronene Adsorbed on Gold, and a Gold Cluster Adsorbed on Graphene: Structural and Electronic Properties. Phys. Rev. B: Condens. Matter Mater. Phys. 2012, 85, 205423.

(30) Rafiee, J.; Mi, X.; Gullapalli, H.; Thomas, A. V.; Yavari, F.; Shi, Y.; Ajayan, P. M.; Koratkar, N. A. Wetting Transparency of Graphene. Nat. Mater. 2012, 11, 217-222.

(31) Robinson, B.; Kay, N.; Kolosov, O. Nanoscale Interfacial Interactions of Graphene with Polar and Non-Polar Liquids. Langmuir 2013, 29, 7735-7742.

(32) Dinelli, F.; Castell, M. R.; Ritchie, D. A.; Mason, N. J.; Briggs, G. A. D.; Kolosov, O. V. Mapping Surface Elastic Properties of Stiff and Compliant Materials on the Nanoscale Using Ultrasonic Force Microscopy. Philos. Mag. A 2000, 80, 2299-2323.

(33) Dinelli, F.; Biswas, S. K.; Briggs, G. A. D.; Kolosov, O. V. Ultrasound Induced Lubricity in Microscopic Contact. Appl. Phys. Lett. 1997, 71, 1177-1179.

(34) Dinelli, F.; Assender, H. E.; Takeda, N.; Briggs, G. A. D.; Kolosov, O. V. Elastic Mapping of Heterogeneous Nanostructures with Ultrasonic Force Microscopy (Ufm). Surf. Interface Anal. 1999, 27, 562-567.

(35) Tinker-Mill, C.; Mayes, J.; Allsop, D.; Kolosov, O. Ultrasonic Force Microscopy for Nanomechanical Characterization of Early and Late-Stage Amyloid-B Peptide Aggregation. Sci. Rep. 2014, 4, 4004.

(36) Yamazaki, K.; Kunii, S.; Ogino, T. Characterization of Interfaces between Graphene Films and Support Substrates by Observation of Lipid Membrane Formation. J. Phys. Chem. C 2013, 117, 1891318918.

(37) Zenasni, A.; Delamoreanu, A.; Rabot, C. Free-Suspended Graphene Synthesis Via Carbon Diffusion through Platinum-Based Metal. Appl. Phys. Lett. 2012, 100, 151907.

(38) Mazzocco, R.; Robinson, B.; Dickinson, J.; Boxall, C.; Kolosov, O. In Dynamic Mesoscale Interfacial Characterisation of Graphene and Graphene Oxide Thin Films in Water, Dodecane and Their Vapour Using Qcm, NSTI-Nanotech 2012; CRC Press - Taylor and Francis Group: Santa Clara, USA, 2012; pp 173-176.

(39) Robinson, B. J.; Kolosov, O. V. Probing Nanoscale GrapheneLiquid Interfacial Interactions Via Ultrasonic Force Spectroscopy. Nanoscale 2014, 6, 10806-10816.

(40) Kolosov, O.; Yamanaka, K. Nonlinear Detection of Ultrasonic Vibrations in an Atomic-Force Microscope. Jpn. J. Appl. Phys. Part 2 Lett. 1993, 32, L1095-L1098.

(41) Giessibl, F. J. High-Speed Force Sensor for Force Microscopy and Profilometry Utilizing a Quartz Tuning Fork. Appl. Phys. Lett. 1998, 73, 3956-3958.

(42) Todorov, I. T.; Smith, W.; Trachenko, K.; Dove, M. T. Dl_Poly_3: New Dimensions in Molecular Dynamics Simulations Via Massive Parallelism. J. Mater. Chem. 2006, 16, 1911-1918.

(43) Mayo, S. L.; Olafson, B. D.; Goddard, W. A. Dreiding: A Generic Force Field for Molecular Simulations. J. Phys. Chem. 1990, 94, 8897-8909.

(44) Soler, J. M.; Artacho, E.; Gale, J. D.; García, A.; Junquera, J.; Ordejón, P.; Sánchez-Portal, P. The Siesta Method for Ab Initio Order- N Materials Simulation. J. Phys.: Condens. Matter 2002, 14, 2745.

(45) Dion, M.; Rydberg, H.; Schröder, E.; Langreth, D. C.; Lundqvist, B. I. Van Der Waals Density Functional for General Geometries. Phys. Rev. Lett. 2004, 92, 246401. 
(46) Dion, M.; Rydberg, H.; Schröder, E.; Langreth, D. C.; Lundqvist, B. I. Erratum: Van Der Waals Density Functional for General Geometries [Phys. Rev. Lett. 92, 246401 (2004)]. Phys. Rev. Lett. 2005, 95, 109902.

(47) Langreth, D. C.; Dion, M.; Rydberg, H.; Schröder, E.; Hyldgaard, P.; Lundqvist, B. I. Van Der Waals Density Functional Theory with Applications. Int. J. Quantum Chem. 2005, 101, 599-610. 